\title{
STONE MV-ALGEBRAS AND STRONGLY COMPLETE MV-ALGEBRAS
}

\author{
JEAN B. NGANOU
}

\begin{abstract}
The article has two main objectives: characterize compact Hausdorff topological MV-algebras and Stone MV-algebras on one hand, and characterize strongly complete MV-algebras on the other hand. We obtain that compact Hausdorff topological MV-algebras are product (both topological and algebraic) of copies $[0,1]$ with the interval topology and finite Lukasiewicz chains with discrete topology. Going one step further we also prove that Stone MV-algebras are product (both topological and algebraic) of finite Lukasiewicz chains with discrete topology. In the second part we prove that an MV-algebra is isomorphic to its profinite completion if and only if it is profinite and its only maximal ideals of finite ranks are principal.
\end{abstract}

To the memory of a great mentor, John V. Leahy (1937-2015).

\section{INTRODUCTION}

An MV-algebra can be defined as an Abelian monoid $(A, \oplus, 0)$ with an involution $\neg: A \rightarrow A$ (i.e., $\neg \neg x=x$ for all $x \in A$ ) satisfying the following axioms for all $x, y \in A: \neg 0 \oplus x=\neg 0, \neg(\neg x \oplus y) \oplus y=\neg(\neg y \oplus x) \oplus x$. For any $x, y \in A$, if one writes $x \leq y$ when $\neg x \oplus y=\neg 0:=1$, then $\leq$ induces a partial order on $A$, which is in fact a distributive lattice order where $x \vee y=\neg(\neg x \oplus y) \oplus y$ and $x \wedge y=\neg(\neg x \vee \neg y)$. MV-algebras form an equational class that contains the variety of Boolean algebras (as MV-algebras satisfying $x \wedge \neg x=0)$. An ideal of an MV-algebra is a nonempty subset $I$ of $A$ such that (i) for all $x, y \in I, x \oplus y \in I$ and (ii) for all $x \in A$ and $y \in I, x \leq y$ implies $x \in I$. A prime ideal of $A$ is proper ideal $P$ such that whenever $x \wedge y \in P$ with $x, y \in A$, then $x \in P$ or $y \in P$. Maximal ideal has the usual meaning.

2010 Mathematics Subject Classification. 06D35, 06E15, 06D50

Key words: topological MV-algebra, Stone MV-algebra, zero-dimensional, profinite MValgebra.

August 24, 2018. 
A topological MV-algebra is an MV-algebra $(A, \oplus, \neg, 0)$ together with a topology $\tau$ such that $\oplus$ and $\neg$ (and in particular $\vee, \wedge$ ) are $\tau$-continuous. A detailed treatment of topological MV-algebras, a category that is clearly a subcategory of topological lattices can be found in [12]. A topological MValgebra is called a Stone MV-algebra if its topology is Stone (compact, Hausdorff, and zero-dimensional). The prototype of (topological) MV-algebra is the unit real interval $[0,1]$ equipped with the operation of truncated addition $x \oplus y=\min \{x+y, 1\}$, negation $\neg x=1-x$, and the identity element 0 . For each integer $n \geq 2, \mathrm{E}_{n}=[0,1] \cap \mathbb{Z} \frac{1}{n-1}$ is an MV-subalgebra of $[0,1]$, (the Łukasiewicz chain with $n$ elements), and up to isomorphism every nontrivial finite MV-chain is of this form. Each $\mathrm{E}_{n}(n \geq 2)$ is clearly a Stone MV-algebra under the discrete topology.

A great deal of research on topological lattices has gravitated around the following important problems.

Problem 1.1. An algebra is profinite if it is the inverse limit of an inverse system of finite algebras. Since the inverse limit of an inverse family of finite discrete spaces is a Stone space, each profinite algebra is naturally a Stone topological algebra. A question of interest that arises is: for what algebraic categories $\mathbb{A}$ is the converse true?, that is every Stone $\mathbb{A}$-algebra is profinite?

Problem 1.2. For what algebraic categories $\mathbb{A}$ is zero-dimensionality automatic for Stone $\mathbb{A}$-algebras? In other words, what algebraic categories $\mathbb{A}$ satisfy: an $\mathbb{A}$-algebra is Stone if and only if it is compact and Hausdorff?

Most of [13, Section VI.2.6] is devoted to investigating problem [1.1. It is shown that the categories of Boolean algebras, of distributive lattices, of Heyting algebras all satisfy the property stated in problem 1.1.

As for problem 1.2, the category of Boolean algebras is most likely the best known example. Indeed, a result of Strauss [16] states that a Boolean algebra admits a Stone topology if and only if it admits a compact and Hausdorff topology if and only if it is a power set algebra. Other well-known categories with this property include the category of orthomodular lattices [8, Lemma 3], and the category of Heyting algebras [6]. A new and simpler proof of Strauss's result can be found in [5].

During the author's BLAST 2014's presentation on "profinite MV-algebras and multisets" [15], several attendees asked about the position of the category of MV-algebras with respect to the above problems. The primary goal of the present paper is to provide a complete answer to those questions and study related problems. 
With respect to problem 1.1, we obtain that the category of MV-algebras is another is another category with the stated property. Indeed, we prove that an MV-algebra admits a Stone topology if and only if it is profinite if and only if it is the product of finite Łukasiewicz chains.

With respect to problem 1.2 , since $[0,1]$ with the interval topology is clearly a compact Hausdorff topological MV-algebra (and this is the only topology making $[0,1]$ a topological MV-algebra), which is not zero-dimensional, then the category of MV-algebras does not have the property of problem 1.2. Indeed, we obtain that MV-algebras admitting compact Hausdorff topologies are product of copies $[0,1]$ and finite Łukasiewicz chains.

In the final section of the article, we consider the problem of MV-algebras that are isomorphic to their profinite completions. This question has been answered for Boolean algebras and Heyting algebras. Indeed, it is known that a Boolean algebra is isomorphic to its profinite completion if and only if it is finite (see for e.g., comment after the proof of [4, Theorem 4.6]), while a Heyting algebra $H$ is isomorphic to its profinite completion if and only if it is finitely approximable, complete, and the kernel of every finite homomorphic image of $H$ is a principal filter of $H$ [4, Theorem 3.10]. For MV-algebras, we prove that an MV-algebra whose all maximal ideals have finite ranks is isomorphic to its profinite completion (called here strongly complete) if and only if it is finite. It is also proved that a general MV-algebra is strongly complete if and only if it is profinite and all its maximal ideals of finite ranks are principal.

For basic MV-algebras terminologies, the reader may refer to [10].

\section{Compact Hausdorff topological MV-algebras}

We recall that the interval topology on any poset $(A, \leq)$ is the smallest topology for which all the sets of the form

$$
[a, b]:=\{x \in A: a \leq x \leq b\}
$$

$(a, b \in A, a \leq b)$ are closed. It is also known that the interval topology of any complete distributive complete lattice is compact and Hausdorff (see e.g., [13, Corollary VI.1.11]). In particular, the interval topology of any complete chain is compact and Hausdorff, and is indeed by [13, Theorem VII.1.9], the only compact Hausdorff lattice topology on the chain. In addition, compact topological MV-algebras are complete (see, e.g., [12, Theorem 3.41]), and (up to isomorphism) the only complete MV-chains are $[0,1]$ and $\mathrm{Ł}_{n}$ for some $n \geq 2$. It follows that up to homeomorphism, the only compact Hausdorff topological 
MV-chains are the unit interval with the interval topology and finite MVchains with the discrete topologies. In particular, the interval topology is the only compact Hausdorff topology on the unit interval $[0,1]$ with respect to which the functions $(x, y) \mapsto \min (x+y, 1)$ and $x \mapsto 1-x$ are continuous.

Lemma 2.1. Let $A=\prod_{x \in X} A_{x}$, where $A_{x}=[0,1]$ or $A_{x}$ is a finite $M V$-chain. Then, the only compact Hausdorff topology on $A$ that makes $A$ a topological $M V$-algebra is the natural product topology. That is the product topology, where each copy of $[0,1]$ carries the interval topology, while each finite $M V$-chain has discrete topology.

Proof. Let $\mathfrak{p}$ be the product topology on $A$, where each copy of $[0,1]$ carries the interval topology, while each finite MV-chain has discrete topology. Then, $\mathfrak{p}$ is a compact Hausdorff topology on $A$ making it a topological MV-algebra (in particular a topological lattice). It follows from [13, Theorem VII.1.9] that $\mathfrak{p}$ is the only compact Hausdorff topology on $A$ that makes $A$ a topological MV-algebra.

Note that if $A=\prod_{x \in X} A_{x}$, where $A_{x}=[0,1]$ or $A_{x}$ is a finite MV-chain, then $A$ is complete and completely distributive. Therefore, the interval topology on $A$ is a compact Hausdorff topology on $A$ that makes $A$ a topological MValgebra. It follows from the Lemma that the product topology on $A$ coincides with the interval topology.

Next, we characterize all compact Hausdorff MV-algebras.

Theorem 2.2. For every $M V$-algebra $A$, the following assertions are equivalent:

1. A admits a topology making it a compact and Hausdorff topological $M V$-algebra;

2. A is complete and completely distributive;

3. A is isomorphic to $\prod_{x \in X} A_{x}$, where $A_{x}=[0,1]$ or $A_{x}$ is a finite $M V$ chain.

4. A is isomorphic to a closed $M V$-subalgebra of an $M V$-algebra of the form $[0,1]^{X}$.

Proof. 1. $\Rightarrow 2$. Suppose that $A$ admits a topology making it a compact and Hausdorff topological MV-algebra. Consider the Boolean center $B(A)$ of $A$ equipped with the subspace topology of $A$. Recall that $B(A)=\{a \in A$ : $a \wedge \neg a=0\}=f^{-1}(\{0\})$, where $f: A \rightarrow A$ is the map defined by $f(a)=$ $a \wedge \neg a$. Since $f$ is clearly continuous and $\{0\}$ is closed, then $B(A)$ is a closed subspace of the compact space $A$. Therefore, $B(A)$ admits a compact and 
Hausdorff topological making it a topological Boolean algebra. Consequently, $B(A)$ is a power set algebra as proved by Strauss [16], in particular, $B(A)$ is atomic. In addition, since compact Hausdorff MV-algebras are complete (see e.g., [12, Theorem 3.41]), then $A$ is complete and by [10, Proposition 6.8.1] or [9, Theorem 2.6], $A$ is complete and completely distributive.

2. $\Rightarrow 3$. Suppose that $A$ is complete and completely distributive. Then, by [10, Proposition 6.8.1] $A$ is the direct product of complete MV-chains. But (up to isomorphism), the only complete MV-chains are finite MV-chain and $[0,1]$.

3. $\Rightarrow$ 4. Let $S=: \prod_{x \in X} A_{x}$, where $A_{x}=[0,1]$ or $A_{x}$ is a finite MV-chain. Then $A$ is isomorphic to $S$, which is clearly a closed MV-subalgebra of an MV-algebra of the form $[0,1]^{X}$.

4. $\Rightarrow 1$. $A$ is isomorphic to a closed MV-subalgebra $S$ of an MV-algebra of the form $[0,1]^{X}$. Then, with the subspace topology, $S$ is a compact Hausdorff topological MV-algebra. Consider the topology

It follows from Theorem 2.2 that any two compact Hausdorff topological MV-algebras that are isomorphic are automatically homeomorphic.

When zero-dimensionality is added to the characterization of Theorem 2.2 , one obtains a simple characterization of Stone MV-algebras. First, we observe that $[0,1] \times \mathrm{E}_{2}$ is not a Stone MV-algebra. Indeed, $[0,1] \times\{0\}$ is a connected subset of $[0,1] \times \mathrm{E}_{2}$, which is not a singleton. Therefore, $[0,1] \times \mathrm{E}_{2}$ is not totally disconnected.

Theorem 2.3. For every $M V$-algebra A, the following assertions are equivalent:

1. A admits a topology making it a Stone topological MV-algebra;

2. A admits a topology making it a Stone topological lattice;

3. A is isomorphic to $\prod_{x \in X} A_{x}$, where each $A_{x}$ is a finite $M V$-chain;

4. A admits a topology making it a compact Hausdorff topological $M V$ algebra and $[0, a]$ is a finite $M V$-chain for all atoms a of $B(A)$;

5. A is profinite.

Proof. 1. $\Rightarrow 2$. This is clear.

2 . $\Rightarrow 3$. Suppose that $A$ admits a topology $\tau$ making it a Stone topological lattice. Then as in the proof of Theorem [2.2, we obtain that the Boolean center $B(A)$ of $A$ admits a topology (the subspace topology) that makes it a Stone topological lattice. It follows from [13, Proposition VII.1.16] that $B(A)$ is complete and atomic. Since $A$ is compact, then $A$ is complete. Then, by [10, Proposition 6.8.1] $A$ is the direct product of complete MV-chains. But (up to isomorphism), the only complete MV-chains are finite MV-chain and 
$[0,1]$. Hence, $A$ is isomorphic to $\prod_{x \in X} A_{x}$, where $A_{x}=[0,1]$ or $A_{x}$ is a finite MV-chain. We claim that there is no $x \in X$ such that $A_{x}=[0,1]$. First, since $\tau$ is a compact and Hausdorff topology on $A$, then by Lemma 2.1 the topology of $\tau$ is the natural product topology, which is the same as the interval topology. Now, if there were an $x_{0} \in X$ such that $A_{x_{0}}=[0,1]$, then $A$ would contain a closed MV-subalgebra isomorphic to $[0,1] \times \mathrm{E}_{2}$. Indeed, let $I=\left\{f \in A: f(x)=0\right.$, for all $\left.x \neq x_{0}\right\}$, then $I$ is clearly a closed ideal of $A$, and $S:=I \cup \neg I$ is the MV-subalgebra of $A$ generated by $I$ (see e.g., [?, Lem. 2.7.5]). Finally, $S$ is clearly closed ( $\neg$ is closed map) and is isomorphic to $[0,1] \times \mathrm{E}_{2}$. As closed MV-subalgebra of a Stone MV-algebra, $S$ would be a Stone MV-algebra, which would imply that $[0,1] \times \mathrm{E}_{2}$ is a Stone MV-algebra. This would contradict the comments preceding the theorem. Thus, each $A_{x}$ is a finite MV-chain and $A$ is isomorphic to $\prod_{x \in X} A_{x}$.

3. $\Rightarrow 4$. Suppose $A$ is isomorphic to $\prod_{x \in X} A_{x}$, where each $A_{x}$ is a finite MVchain. Then equipping each $A_{x}$ with the discrete topology, and considering the product topology on $A, A$ is clearly compact Hausdorff topological MValgebra. In addition $B(A) \cong 2^{X}$ and it follows that for every atom $a$ of $B(A)$, $[0, a] \cong A_{x}$, which is a finite MV-chain.

4 . $\Rightarrow 3$. Suppose that $A$ admits a topology making it a compact Hausdorff topological MV-algebra and $[0, a]$ is a finite MV-chain for all atoms $a$ of $B(A)$. By Theorem 2.2, $A$ is isomorphic to $\prod_{x \in X} A_{x}$, where $A_{x}=[0,1]$ or $A_{x}$ is a finite MV-chain. For each $x \in X$, consider the element $\alpha_{x} \in A$ defined by $\alpha_{x}(t)=0$ if $t \neq x$ and $\alpha_{x}(x)=1$. Then for each $x \in X, \alpha_{x} \in B(A)$ and $\left[0, \alpha_{x}\right] \cong A_{x}$. Therefore, $A_{x}$ is a finite MV-chain, for all $x \in X$.

3 . $\Leftrightarrow 5$. This is part of [15, Theorem 2.5]. Finally, profinite algebras are always Stone algebras, hence $5 . \Rightarrow 1$.

Note that if $A$ is a profinite MV-algebra, then $A \cong \prod_{x \in X}[0, x]$, where $X$ is the set of atoms of $B(A)$.

\section{Strongly Complete MV-Algebras}

An MV-algebra will be called strongly complet 11 if it is isomorphic to its profinite completion. We start by recalling the construction of the profinite completion for MV-algebras. Given an MV-algebra $A$ and an ideal $I$ of $A$, the relation $\equiv_{I}$ defined on $A$ by $x \equiv_{I} y$ if and only if $\neg(\neg x \oplus y) \oplus \neg(x \oplus \neg y) \in I$, is a congruence on $A$. The quotient MV-algebra induced by this congruence is denoted by $A / I$, and the congruence class of $a \in A$ denoted by $[a]_{I}$. Let $A$ be an MV-algebra and let $\operatorname{id}_{f}(A)$ be the set of all ideals $I$ of $A$ such that $A / I$

\footnotetext{
${ }^{1}$ This terminology is borrowed from the well-known theory of profinite groups.
} 
is finite. For every $I, J \in \operatorname{id}_{f}(A)$ such that $I \subseteq J$, let $\phi_{J I}: A / I \rightarrow A / J$ be the natural homomorphism, i.e., $\phi_{J I}\left([a]_{I}\right)=[a]_{J}$ for all $a \in A$. Observe that $\left.\operatorname{id}_{f}(A), \supseteq\right)$ is a directed set, and $\left\{\operatorname{id}_{f}(A),\{A / I\},\left\{\phi_{J I}\right\}\right\}$ is an inverse system of MV-algebras. The inverse limit of this inverse system is called the profinite completion of the MV-algebra $A$, and commonly denoted by $\widehat{A}$. The following description of $\widehat{A}$ is also well known:

$$
\widehat{A} \cong\left\{\alpha \in \prod_{I \in \operatorname{id}_{f}(A)} A / I: \phi_{J I}(\alpha(I))=\alpha(J) \text { whenever } I \subseteq J\right\}
$$

In the sequel, the set of prime ideals of $A$ will be denoted by $\operatorname{Spec}(A)$ and is endowed with the Zariski's topology. The set $\operatorname{Max}(A)$ of maximal ideals of $A$ inherits the subspace topology of $\operatorname{Spec}(A)$.

We have the following characterization of $\operatorname{id}_{f}(A)$.

Proposition 3.1. Let $A$ be an $M V$-algebra and $I$ be any ideal of $A$. Then $I \in i d_{f}(A)$ if and only if there exist $M_{1}, M_{2}, \ldots, M_{r} \in \operatorname{Max}(A) \cap i d_{f}(A)$ such that $I=M_{1} \cap M_{2} \cap \ldots \cap M_{r}$.

Proof. Suppose that $I$ is an ideal of $A$, with $A / I$ finite. Then, by [10, Proposition 3.6.5], there is an isomorphism $\varphi: A / I \rightarrow \prod_{i=1}^{r} \mathrm{E}_{n_{i}}$, for some integers $n_{1}, n_{2}, \ldots, n_{r} \geq 2$. For each $k=1,2, \ldots, r$, let $M_{k}=\operatorname{ker}\left(q_{k} \circ \varphi \circ p_{I}\right)$, where $q_{k}$ is the natural projection $\prod_{i=1}^{r} \mathrm{E}_{n_{i}} \rightarrow \mathrm{E}_{k}$ and $p_{I}: A \rightarrow A / I$ is the canonical projection. Then $M_{k}$ is a maximal ideal of $A$ since $A / M_{k} \cong \mathrm{E}_{k}$, which is simple. In addition, it is clear that $I=M_{1} \cap M_{2} \cap \ldots \cap M_{r}$.

Conversely, suppose there exist $M_{1}, M_{2}, \ldots, M_{r} \in \operatorname{Max}(A) \cap \operatorname{id}_{f}(A)$ such that $I=M_{1} \cap M_{2} \cap \ldots \cap M_{r}$. Consider the homomorphism $\varphi: A \rightarrow \prod_{i=1}^{r} A / M_{i}$ defined by $\varphi(a)=\left([a]_{M_{i}}\right)_{i}$. Then, $\operatorname{ker} \varphi=I$ and $A / I$ is isomorphic to a sub-MV-algebra of $\prod_{i=1}^{r} A / M_{i}$. Hence, $I \in \operatorname{id}_{f}(A)$.

Recall [11] that a maximal ideal $M$ of an MV-algebra $A$ is said to have a finite rank $n$, for some integer $n \geq 2$ if $A / M \cong \mathrm{E}_{n}$, otherwise one says that $M$ has infinite rank. One should observe that every maximal ideal of a Boolean algebra has finite rank. An MV-algebra $A$ is called regular [3] if for every prime ideal $N$ of its Boolean center $B(A)$, the ideal of $A$ generated by $N$ is a prime ideal of $A$. It is known [3, Proposition 26] that if $A$ is a regular MV-algebra, then the map $P \mapsto P \cap B(A)$ defines a homeomorphism from $\operatorname{Max}(A)$ onto $\operatorname{Max}(B(A))=\operatorname{Spec}(B(A))$.

Theorem 3.2. Let $A$ be a regular $M V$-algebra in which every maximal ideal has finite rank. 
1. Then the map $\Psi: i d_{f}(A) \rightarrow i d_{f}(B(A))$ defined by $\Psi(I)=I \cap B(A)$ is an inclusion preserving and reversing bijection.

2. For every $I \in i d_{f}(A)$, the map $\Theta_{I}: B(A) / \Psi(I) \rightarrow B(A / I)$ defined by $\Theta_{I}([a])=[a]_{I}$ is an isomorphism.

3. For every $I \subseteq J$ in $i d_{f}(A)$, if $\phi_{J I}: A / I \rightarrow A / J$ is the transition homomorphism, the following diagram is commutative.

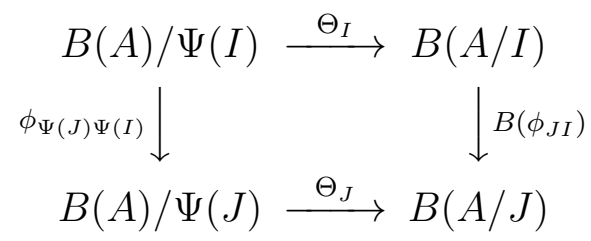

Proof. 1. Since every maximal ideal of $A$ (resp. $B(A))$ has finite rank, it follows from Proposition 3.1 elements of $\operatorname{id}_{f}(A)$ (resp. $\left.\operatorname{id}_{f}(B(A))\right)$ are simply finite intersections of maximal ideals of $A$ (resp. $B(A)$ ). Therefore, from the observation before the Proposition, we obtain that $\Psi$ is well-defined. To prove that $\Psi$ is bijective, we construct its inverse. Indeed, consider $\Phi: \operatorname{id}_{f}(B(A)) \rightarrow$ $\operatorname{id}_{f}(A)$ defined as follows. Let $V \in \operatorname{id}_{f}(A)$, then $V=V_{1} \cap \ldots \cap V_{s}$, where each $S_{i}$ is a maximal ideal of $B(A)$. By [3, Proposition 26], there exists a unique set of maximal ideals $M_{1}, \ldots, M_{s}$ of $A$ such that $V_{i}=M_{i} \cap B(A)$ for each $i=1, \ldots, s$. Consider $I=M_{1} \cap \ldots \cap M_{s}$, which is in $\operatorname{id}_{f}(A)$. It is clear that $\Psi$ and $\Phi$ are inverses of each other. Clearly $\Psi$ preserves the inclusion. For the inclusion reversing, suppose that $I \cap B(A) \subseteq J \cap B(A)$, then $I \cap J \cap B(A)=I \cap B(A)$. But, since $\Psi$ is one-to-one, then $I \cap J=I$ and $I \subseteq J$.

2. It is clear that the map $a \mapsto[a]_{I}$ is a homomorphism from $B(A)$ to $B(A / I)$, whose kernel is $\Psi(I)$. Therefore, $\Theta_{I}$ is an injective homomorphism. But, since $B(A) / \Psi(I)$ and $B(A / I)$ are finite Boolean algebras, then $\Theta_{I}$ is an isomorphism as claimed.

3. This follows from the definitions of the various homomorphisms.

It is worth pointing out that the class of MV-algebras covered by Theorem 3.2 is quite large. Indeed, we have the following well-known two subclasses:

(a) Let $B$ be a profinite Boolean algebra. Then $B$ is a regular MV-algebra in which every maximal ideal has rank 2. This is special case of Proposition 4.4 below.

(b) Every non-simple MV-chain of finite rank (see [10, Section 8.3]) is a regular MV-algebra in which every maximal ideal has finite rank. In fact, any such MV-chain $C$ has a unique maximal ideal whose rank is the same as that of $C$. 
Proposition 3.3. Let $A$ be a regular $M V$-algebra in which every maximal ideal has finite rank. Then,

$$
B(\widehat{A}) \cong \widehat{B(A)}
$$

Proof. We have the following sequence of isomorphisms. The first one is from the standard description of the profinite completion. The second follows from Theorem 3.2 (1). The third follows from Theorem 3.2(2-3) and the remaining isomorphisms follow from the definition of the Boolean center.

$$
\begin{aligned}
\widehat{B(A)} & \cong\left\{\alpha \in \prod_{V \in \operatorname{id}_{f}(B(A))} B(A) / V: \phi_{U V}(\alpha(V))=\alpha(U) \text { whenever } V \subseteq U\right\} \\
& \cong\left\{\alpha \in \prod_{\Psi(I) \in \operatorname{id}_{f}(B(A))} B(A) / \Psi(I): \phi_{\Psi(J) \Psi(I)}(\alpha(\Psi(I))=\alpha(\Psi(J)) \text { whenever } I \subseteq J\}\right. \\
& \cong\left\{\alpha \in \prod_{I \in \operatorname{id}_{f}(A)} B(A / I): \phi_{J I}(\alpha(I))=\alpha(J) \text { whenever } I \subseteq J\right\} \\
& \cong\left\{\alpha \in B\left(\prod_{I \in \operatorname{id}_{f}(A)} A / I\right): \phi_{J I}(\alpha(I))=\alpha(J) \text { whenever } I \subseteq J\right\} \\
& \cong B\left(\left\{\alpha \in \prod_{I \in \operatorname{id}_{f}(A)} A / I: \phi_{J I}(\alpha(I))=\alpha(J) \text { whenever } I \subseteq J\right\}\right) \\
& \cong B(\widehat{A})
\end{aligned}
$$

Corollary 3.4. Let $A$ be an $M V$-algebra in which every maximal ideal has finite rank. Then $A$ is strongly complete if and only if $A$ is finite.

Proof. It is clear that finite MV-algebras are strongly complete. Conversely, suppose that $A$ is strongly complete. Then $\widehat{A} \cong A$. Then, since $\widehat{A}$ is profinite, so is $A$. By [15, Theorem 2.5], $A \cong \prod_{x \in X} \mathrm{\iota}_{n_{x}}$ for some set $X$, and some integers $n_{x} \geq 2$. Thus, by [3, Proposition 36, 37], $A$ is regular. Hence, by Proposition $3.3, \widehat{B(A)} \cong B(\widehat{A}) \cong B(A)$. Hence, the Boolean center $B(A)$ of $A$ is strongly complete. But the only strongly complete Boolean algebras are finite ones. Thus, $B(A) \cong 2^{X}$ is finite. Therefore, $X$ is finite and $A$ is finite as needed.

Proposition 3.5. Let $A$ be a strongly complete $M V$-algebra. Then $A$ is profinite and every maximal ideal of $A$ of finite rank is principal. 
Proof. Suppose that $A$ is an MV-algebra isomorphic to its profinite completion $\widehat{A}$. Then, $A$ is profinite since $\widehat{A}$ is profinite by definition. Let $M$ be a maximal of $A$ of finite rank $n$. Then, $M$ can be viewed as an ideal of $\widehat{A}$ of finite rank. Since $\widehat{A}=\left\{\alpha \in \prod_{I \in \operatorname{id}_{f}(A)} A / I: \phi_{J I}(\alpha(I))=\alpha(J)\right.$ whenever $\left.I \subseteq J\right\}$, then $M$ (viewed as an ideal of $\widehat{A}$ ) is equal to $A_{M}:=\{\alpha \in \widehat{A}: \alpha(M)=M\}=\{\alpha \in$ $\left.\prod_{I \in \operatorname{id}_{f}(A)} A / I: \alpha(M)=M\right\} \cap \widehat{A}=\operatorname{ker} p_{M}$, where $p_{M}$ is the natural projection $\widehat{A} \rightarrow A / M$. But, $A_{M}$ is a maximal ideal of $\widehat{A}$ since $\widehat{A} / A_{M} \cong A / M$, which is simple. In addition, $A_{M}$ is clearly clopen in $\widehat{A}$ under the induced topology of $\prod_{I \in \operatorname{id}_{f}(A)} A / I$. Hence, by [15, Lemma 3.1] $A_{M}$ is principal in $\widehat{A}$, and $M$ is principal in $A$.

Recall [15, Theorem 2.5] that if $A$ is a profinite MV-algebra, then $A \cong$ $\prod_{x \in X} \mathrm{E}_{n_{x}}$ for some set $X$ and $n_{x} \geq 2$ are integers. Let $\mathcal{P}_{f}(X)$ be the set of finite subsets of $X$. For each $S \in \mathcal{P}_{f}(X)$, let $A_{S}:=\prod_{x \in S} \mathrm{七}_{n_{x}}$. Then for $S \subseteq T$ in $\mathcal{P}_{f}(X)$, there exists a natural homomorphism $\phi_{S T}: A_{T} \rightarrow A_{S}$ defined by $\phi_{S T}(\alpha)(x)=\alpha(x)$ for all $\alpha \in A_{T}$ and $x \in S$. It is easily verified that $\left(\mathcal{P}_{f}(X), \supseteq\right)$ is a directed set and $\left\{\left\{\mathcal{P}_{f}(X)\right\},\left\{A_{S}\right\}_{S},\left\{\phi_{S T}\right\}\right\}$ is an inverse system. Let

$$
\widetilde{A}:=\lim _{\longleftarrow}\left\{\left\{\mathcal{P}_{f}(X)\right\},\left\{A_{S}\right\}_{S},\left\{\phi_{S T}\right\}\right\}
$$

Lemma 3.6. Let $A$ be a profinite $M V$-algebra such that every maximal ideal of $A$ of finite rank is principal. Then,

$$
\widehat{A} \cong \widetilde{A}
$$

Proof. It is enough to prove that the inverse systems $\left\{\operatorname{id}_{f}(A),\{A / I\},\left\{\phi_{J I}\right\}\right\}$ and $\left\{\left\{\mathcal{P}_{f}(X)\right\},\left\{A_{S}\right\}_{S},\left\{\phi_{S T}\right\}\right\}$ are isomorphic. Consider the map $\Theta: \mathcal{P}_{f}(X) \rightarrow$ $\operatorname{id}_{f}(A)$ defined by $\Theta(S)=\cap_{x \in S} M_{x}$, where $M_{x}$ is the kernel of the natural projection $p_{x}: A \rightarrow \mathrm{E}_{n_{x}}$. It follows from Proposition 3.1, the fact every maximal ideal of $A$ of finite rank is principal and [15, Proposition 3.1] that $\Theta$ is a inclusion-preserving bijection. In addition, for all $S \in \mathcal{P}_{f}(X)$, there is a canonical isomorphism $\varphi_{S}: A_{S} \rightarrow A / \Theta(S)$. To see that the systems $\left\{\operatorname{id}_{f}(A),\{A / I\},\left\{\phi_{J I}\right\}\right\}$ and $\left\{\left\{\mathcal{P}_{f}(X)\right\},\left\{A_{S}\right\}_{S},\left\{\phi_{S T}\right\}\right\}$ are isomorphic, one needs to verify that for all $S \subseteq T$, the following diagram is commutative.

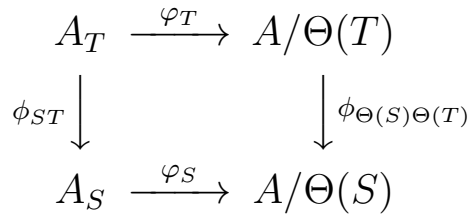

But, this is a simple verification. 
Theorem 3.7. Let $A$ be a profinite $M V$-algebra such that every maximal ideal of $A$ of finite rank is principal. Then $A$ is strongly complete.

Proof. Recall that $\widetilde{A} \cong\left\{f \in \prod_{S \in \mathcal{P}_{f}(X)} A_{S}: \phi_{S T}(f(T))=f(S)\right.$, whenever $\left.S \subseteq T\right\}$.

Since $\widehat{A} \cong \widetilde{A}$ from Lemma 3.6, we shall prove that $A$ is isomorphic to $\widetilde{A}$. For $f \in A$, define $\varphi(f) \in \prod_{S \in \mathcal{P}_{f}(X)} A_{S}$ by $\varphi(f)(S)(x)=f(x)$ for all $S \in \mathcal{P}_{f}(X)$ and all $x \in S$. We claim that $\varphi(f) \in \widetilde{A}$. Suppose $S \subseteq T$, and $x \in S$, then $\phi_{S T}(\varphi(f)(T))(x)=\varphi(f)(T)(x)=f(x)=\varphi(f)(S)(x)$. Therefore, $\varphi(f) \in \widetilde{A}$ and $\varphi$ is a well-defined map from $A \rightarrow \widetilde{A}$. It is easy to verify that $\varphi(f \oplus g)=$ $\varphi(f) \oplus \varphi(g)$ and $\varphi(\neg f)=\neg \varphi(f)$, and so $\varphi$ is a homomorphism. It remains to prove that $\varphi$ is bijective.

Injectivity: Let $f, g \in A$ such that $\varphi(f)=\varphi(g)$, then for all $x \in X, \varphi(f)(\{x\})(x)=$

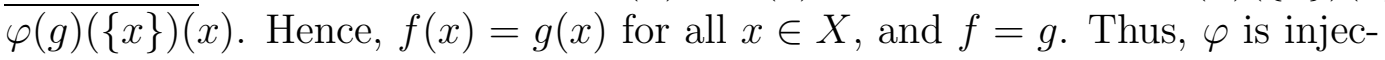
tive.

Surjectivity: Let $\alpha \in \widetilde{A}$, then $\alpha(\{x\}) \in A_{\{x\}}\left(=\prod_{t \in\{x\}} \mathrm{E}_{n_{t}}\right)$. Define $f \in A$ by

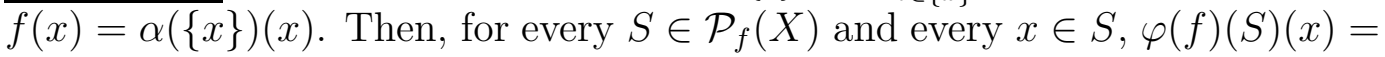
$f(x)=\alpha(\{x\})(x)=\phi_{\{x\} S}(\alpha(S))(x)=\alpha(S)(x)$. Hence, $\varphi(f)=\alpha$, and $\varphi$ is surjective.

Thus, $\varphi$ is an isomorphism as desired.

Combining Proposition 3.5 and Theorem 3.7, we obtain a necessary and sufficient condition for the strong completeness of MV-algebras.

Corollary 3.8. An MV-algebra $A$ is strongly complete if and only if $A$ is profinite and every finite rank maximal ideal of $A$ is principal.

Since every maximal ideal of a Boolean algebra has rank 2, and every infinite profinite Boolean algebra (powerset) has non-principal maximal ideals, then it follows from Corollary 3.8 that the only strongly complete Boolean algebras are the finite ones. This provides another proof of this well-known result.

\section{EXAMPLES}

In this section we construct concrete examples and non-examples of strongly complete MV-algebras. The main tool used here is the well-known connection between the maximal spectrum of profinite and the theory of ultrafilters. Recall that if $A$ is a profinite MV-algebra, then $A \cong \prod_{x \in X} \mathrm{E}_{n_{x}}$ for some set $X$, and some integers $n_{x} \geq 2$. In addition, by [15, Corollary 3.4], the set $\eta(A):=\left\{n_{x}: x \in X\right\}$ is uniquely determined by $A$.

In the remainder of this section $A:=\prod_{x \in X} \mathrm{E}_{n_{x}}$. We shall recall a known description of the maximal spectral space of $A$. It is likely that the presentation 
given of this description is not new, but for lack of direct reference, and also to increase the readability, we include it. For $f \in A$ and $\epsilon>0$,

$$
D(f, \epsilon):=\{x \in X: f(x)<\epsilon
$$

It is easy to see that for all $f, g \in A$ and $\epsilon, \delta>0: D(f \oplus g, \min (\epsilon, \delta)) \subseteq$ $D(f, \epsilon) \cap D(g, \delta)$

Definition 4.1. Let $f \in A, \mathcal{U}$ an ultrafilter of $X$ and $t \in[0,1]$. We say that $f$ converges to $t$ along $\mathcal{U}$ if for every $\epsilon>0$, there exists $S \in \mathcal{U}$ such that $f(S) \subseteq(t-\epsilon, t+\epsilon)$.

Note that this is equivalent to for every $\epsilon>0,\{x \in X:|f(x)-t|<\epsilon\}=$ $f^{-1}(t-\epsilon, t+\epsilon) \in \mathcal{U}$.

Since $[0,1]$ is compact and Hausdorff, then every $f \in A$ has a unique limit along any ultrafilter $\mathcal{U}$ of $X$ (see for e.g., [1, Prop. 1.7]). We denote this limit by

$$
\lim _{\mathcal{U}} f
$$

Note that $f$ converges to 0 along $\mathcal{U}$ if and only if for every $\epsilon>0, D(f, \epsilon) \in \mathcal{U}$. For every ultrafilter $\mathcal{U}$ of $X$, let

$$
M_{\mathcal{U}}:=\left\{f \in A: \lim _{\mathcal{U}} f=0\right\}=\{f \in A: D(f, \epsilon) \in \mathcal{U} \text { for all } \epsilon>0\}
$$

For every maximal ideal $M$ of $A$, let

$$
\mathcal{U}_{M}:=\{S \subseteq X: D(f, \epsilon) \subseteq S \text { for some } f \in M \text { and } \epsilon>0\}
$$

Proposition 4.2. For every ultrafilter $\mathcal{U}$ of $X$, define the map $\Psi_{\mathcal{U}}: A \rightarrow[0,1]$ by $\Psi_{\mathcal{U}}(f)=\lim _{\mathcal{U}} f$. Then $\Psi$ is an $M V$-homomorphism whose kernel is $M_{\mathcal{U}}$.

Proof. Let $f, g \in A$, and let $\lim _{\mathcal{U}} f=t, \lim _{\mathcal{U}} g=s$. We need to show that $\lim _{\mathcal{U}}(f \oplus g)=t \oplus s$. Let $\epsilon>0$, then since $\oplus:[0,1] \times[0,1] \rightarrow[0,1]$ is continuous, there exists $\delta>0$ such that for all $a, b \in[0,1](|a-t|<\delta$ and $|b-s|<\delta \Rightarrow$ $|a \oplus b-t \oplus s|<\epsilon)$. On the other hand, since $\lim _{\mathcal{U}} f=t$ and $\lim _{\mathcal{U}} g=s$, then $S:=\{x \in X:|f(x)-t|<\delta\} \in \mathcal{U}$ and $T:=\{x \in X:|g(x)-s|<\delta\} \in \mathcal{U}$. But, $S \cap T \subseteq\{x \in X:|f(x) \oplus g(x)-t \oplus s|<\epsilon\}$ and $S \cap T \in \mathcal{U}$, then $\{x \in X:|f(x) \oplus g(x)-t \oplus s|<\epsilon\} \in \mathcal{U}$. Thus, $f \oplus g$ converges to $t \oplus s$ along $\mathcal{U}$. Using the fact that $\neg:[0,1] \rightarrow[0,1]$ is continuous, one can prove in a similar manner that $\lim _{\mathcal{U}} \neg f=\neg \lim _{\mathcal{U}} f$. Hence, $\Psi_{\mathcal{U}}(\neg f)=\neg \Psi_{\mathcal{U}}(f)$ and $\Psi_{\mathcal{U}}(f \oplus g)=\Psi_{\mathcal{U}}(f) \oplus \Psi_{\mathcal{U}}(g)$, for all $f, g \in A$. Therefore, $\Psi$ is an MVhomomorphism. Clearly, from the definition $\operatorname{ker} \Psi_{\mathcal{U}}=M_{\mathcal{U}}$.

The set of maximal ideals of $A$ (in fact any MV-algebra) carries a natural Zariski topology. The resulting space is known as the maximal spectral space of 
$A$ (see e.g., [14, Chap. 4]). Since $A=\prod_{x \in X} \mathrm{E}_{n_{x}}$ is a profinite MV-algebra, it is known that the maximal spectral space of $A$ is the Stone Cech compactification $\beta X$ of $X$ endowed with the discrete topology. More precisely, the following result holds.

Theorem 4.3. The map $M \mapsto \mathcal{U}_{M}$ is a homeomorphism from $\operatorname{Max}(A) \rightarrow \beta X$ whose inverse is $\mathcal{U} \mapsto M_{\mathcal{U}}$.

Note that it follows that every maximal ideals of $A$ are of the form $M_{\mathcal{U}}$, where $\mathcal{U}$ is an ultrafilter of $X$. In addition, $M_{\mathcal{U}}$ is a principal maximal ideal of $A$ if and only if $\mathcal{U}$ is a principal ultrafilter of $X$. Moreover, note that from Proposition 4.2, the quotient $M V$-algebra $A / M_{\mathcal{U}}$ is naturally isomorphic to the sub-MV-algebra of $[0,1]$ of all $\mathcal{U}$-limits of elements of $A$. We can apply this and obtain the following result.

Proposition 4.4. Let $A$ be a profinite $M V$-algebra such that $\eta(A)$ is bounded. Then every maximal ideal of $A$ has finite rank.

Proof. Suppose that $\left\{n_{x}: x \in X\right\}$ has a maximum element, say $n$ and let $M$ be a maximal ideal of $A$. Then, $M=M_{\mathcal{U}}$ for some ultrafilter $\mathcal{U}$ of $X$. We prove that $\operatorname{Im} \Psi_{\mathcal{U}}$ is finite. It is enough to show that for every nonzero $t \in \operatorname{Im} \Psi_{\mathcal{U}}, n t=1$. So, let $f \in A$ such that $\lim _{\mathcal{U}} f \neq 0$, there there exists $\delta_{1}>0$ such that $D\left(f, \delta_{1}\right) \notin \mathcal{U}$. Hence, $X \backslash D\left(f, \delta_{1}\right) \in \mathcal{U}$. We need to show that $\lim _{\mathcal{U}} n f=1$. But contradiction, suppose that $\lim _{\mathcal{U}} n f \neq 1$, then there exists $\delta_{2}>0$ such that $\left\{x \in X:|n f(x)-1|<\delta_{2}\right\} \notin \mathcal{U}$. In particular, note that $\delta_{2}<1$. In addition, since for all $x \in X, f(x)=0$ or $n f(x)=1$, then $\left\{x \in X:|n f(x)-1|<\delta_{2}\right\}=\{x \in X: n f(x)=1\}$. Thus, $Z(f):=\{x \in$ $X: f(x)=0\} \in \mathcal{U}$, since $Z(f)=X \backslash\{x \in X: n f(x)=1\}$. Therefore, $\emptyset=Z(f) \cap\left(X \backslash D\left(f, \delta_{1}\right)\right) \in \mathcal{U}$, which is a contradiction.

It follows from Corollary 3.4 that the only profinite MV-algebras $A$ with bounded $\eta(A)$ are the finite ones.

The next examples illustrate the situation when $\eta(A)$ is unbounded. Unlike Boolean algebras, there exists infinite strongly complete MV-algebras.

Example 4.5. Let $A=\prod_{n=1}^{\infty} \mathrm{E}_{n+1}$. We claim that every non-principal maximal ideal of $A$ has infinite rank. Let $M$ be a non-principal maximal ideal of $A$, then $M=M_{\mathcal{U}}$ for some free ultrafilter $\mathcal{U}$ of $\mathbb{N}$. An application of the Archimedean property of real numbers gives the following fact: For every $0 \leq a<b \leq 1$, there exists a natural number $N$ such that for all integers $n \geq N$, there exists an integer $k_{n}$ satisfying $a \leq k_{n} / n \leq b$. It follows that 
for every $0 \leq a<b \leq 1$, there exists a natural number $N$ and $f \in A$ such that $a \leq f(n) \leq b$ for all integers $n \geq N$. Since $a \leq f(n) \leq b$ for all integers $n \geq N$, then $a \leq \lim _{\mathcal{U}} f \leq b$ as $\mathcal{U}$ is a free ultrafilter. Thus, $\operatorname{Im} \Psi_{\mathcal{U}}$ is dense in $[0,1]$, and in particular $A / M$ is infinite. Note that indeed $A / M \cong[0,1]$ (see for e.g., [14] ).

Not every profinite MV-algebra $A$ with $\eta(A)$ unbounded is strongly complete as the next example shows.

Example 4.6. Let $A=\prod_{k=1}^{\infty} A_{k}$, where $A_{2 k-1}=\mathrm{七}_{2}$ and $A_{2 k}=\mathrm{七}_{2 k}$. Let $S$ be the set of odd natural numbers and $\mathcal{U}$ be any free ultrafilter of $\mathbb{N}$ containing $S$. We claim that $A / M_{\mathcal{U}} \cong \mathrm{E}_{2}$. In fact, suppose that $f \notin M_{\mathcal{U}}$, then there exists $\delta>0$ such that $D(f, \delta) \notin \mathcal{U}$. Hence $\mathbb{N} \backslash D(f, \delta) \in \mathcal{U}$ and $S \cap(\mathbb{N} \backslash D(f, \delta)) \in \mathcal{U}$. But, $S \cap(\mathbb{N} \backslash D(f, \delta))=\{n \in \mathbb{N}: f(n)=1\} \subseteq\{n \in \mathbb{N}:|f(n)-1|<\epsilon\}$ for every $\epsilon>0$. Thus, $\{n \in \mathbb{N}:|f(n)-1|<\epsilon\} \in \mathcal{U}$ for every $\epsilon>0$. Hence $\lim _{\mathcal{U}} f=1$, and $\operatorname{Im} \Psi_{\mathcal{U}}=\mathrm{E}_{2}$. Therefore, $M_{\mathcal{U}}$ is a non-principal maximal ideal of $A$ of finite rank. Whence, $A$ is not strongly complete.

\section{CONCLUSion AND FinAl REMARKS}

In addition to characterizing all compact Haussdorff, and Stone MV-algebras, we have completely characterized the MV-algebras that are isomorphic to their own profinite completions. An important related question (which is a weakening of strong completenes) is about (profinite) MV-algebras that are profinite completions of some MV-algebras. It is known [7, Corollary 5.10] that every profinite Boolean algebra is a profinite completion of some Boolean algebra. It is also known that a profinite bounded distributive lattices that are isomorphic to profinite completions are up to isomorphism the lattices of upsets of representable posets [7, Theorem 5.3]. The same question remains open for Heyting algebras (see comments after the proof of [7, Corollary 5.9]). It is our goal to tackle this question for MV-algebras in an upcoming project.

\section{REFERENCES}

[1] M. A. Alekselev, L. Yu. Glebskii, E. I. Gordon, On Approximation od groups, group actions, and Hopf algebras, J. Math. Sci, 107 , no. 5(2001)4305-4332.

[2] Lee W. Anderson, One dimensional topological lattices, Proc. Amer. Math. Soc. 10 (1959)715-720.

[3] L. P. Belluce, A. Di Nola, S. Sessa, The Prime Spectrum of an MV-algebra, Math. Log. Qart. 40 (1994)331-346.

[4] G. Bezhanishvili, N. Bezhanishvili, Profinite Heyting algebras, Order 25(2008) 211227 . 
[5] G. Bezhanishvili, J. Harding, On the proof that compact Hausdorff Boolean algebras are powersets, preprint.

[6] G. Bezhanishvili, J. Harding, Compact Hausdorff Heyting algebras, preprint.

[7] G. Bezhanishvili, P. J. Morandi, Profinite Heyting algebras and profinite completions of Heyting algebras, Georgian Math. J. 16 (2009)29-47.

[8] T. H. Choe, R. J. Greechie, Profinite orthomodular lattices, Proc. Amer. Math. Soc. 118, no. 4(1993)1053-1060.

[9] R. Cignoli, Complete and atomic algebras of the infinite valued Łukasiewicz logic, Studia Logica 50, no. 3-4(1991) 375-384.

[10] R. Cignoli, I. D'Ottaviano, D. Mundici, Algebraic foundations of many-valued reasoning, Kluwer Academic, Dordrecht(2000).

[11] R. Cignoli, D. Mundici, Stone duality for Dedekind $\sigma$-complete $\ell$-groups with orderunit, J. of Algebra, $\mathbf{3 0 2}$ (2006)848-861

[12] C. S. Hoo, Topological MV-algebras, Topology Appl. 81(1997)103-121.

[13] P. T. Johnstone, Stone spaces, Cambridge University Press, Cambridge (1982).

[14] D. Mundici, Advanced Łukasiewicz calculus and MV-algebras, Trends Log. Stud. Log. Libr., 35, Springer, New York (2011).

[15] J. B. Nganou, Profinite MV-algebras and multisets, Order (2015) DOI 10.1007/s11083-014-9345-5.

[16] D. P. Strauss, Topological lattices, Proc. London Math. Soc. (3)18(1968)217-230.

Department of Mathematics, University of Oregon, Eugene, OR 97403

E-mail address: nganou@uoregon.edu 